\title{
Receptor-selective TRAIL Mutants Target Lymphoid Tumor cells for Apoptosis via TRAIL-R1: Implications for Therapy
}

\author{
Marion MacFarlane $^{1}$, Susan L Kohlhaas, ${ }^{1}$ Michael J Sutcliffe ${ }^{2}$, Martin JS Dyer ${ }^{1}$, Gerald M Cohen ${ }^{1}$ \\ ${ }^{1}$ MRC Toxicology Unit, Hodgkin Building, University of Leicester, Leicester, LE1 9HN. \\ ${ }^{2}$ Manchester Interdisciplinary Biocentre, School of Chemical Engineering and Analytical Science, \\ University of Manchester, Manchester. mm21@le.ac.uk
}

Tumor Necrosis Factor (TNF)-Related Apoptosis-Inducing Ligand (TRAIL/Apo2L) induces apoptosis via triggering of TRAIL-R1 or TRAIL-R2 and activation of caspase-8 within the deathinducing signalling complex (DISC). Importantly, TRAIL been proposed as a novel therapeutic for treating various malignancies, as it selectively induces apoptosis in transformed cells but not in the majority of normal cells. Although many tumour cell lines exhibit marked sensitivity to TRAIL, most primary tumor cells from patients, such as those with chronic lymphocytic leukaemia (CLL), are highly resistant to TRAIL. Previously we have demonstrated that resistance of CLL cells to TRAIL occurs upstream of caspase-8 activation at the level of the TRAIL DISC. However, we have found that exposure to low concentrations of histone deacetylase inhibitors (HDACi) can markedly sensitize CLL cells to TRAIL. These findings prompted us to examine whether the combination of an HDACi and TRAIL could be of therapeutic benefit in CLL.

The ability of different forms of TRAIL, or agonistic mAbs to TRAIL-R1/-R2 (including those currently in Phase I/II clinical trials - mapatumumab/HGS-ETR1 and lexatumumab/HGS-ETR2) to induce apoptosis (assessed by Phosphatidylserine (PS) exposure) was examined in freshly isolated primary B cells from patients with CLL $(n=8)$, in the presence of an HDACi (Depsipeptide). CLL cells were pre-incubated for $16 \mathrm{~h}$, either in medium alone (-) or in the presence $(+)$ of Depsipeptide $(10 \mathrm{nM})$, and then treated for a further $4 \mathrm{~h}$ with different forms of TRAIL $(100 \mathrm{ng} / \mathrm{ml})$, or the agonistic antibodies, ETR1 $(1 \mu \mathrm{g} / \mathrm{ml})$ or ETR2 $(1 \mu \mathrm{g} / \mathrm{ml})$.

Figure 1: TRAIL, combined with an HDACi, targets CLL cells for Apoptosis via TRAIL-R1.

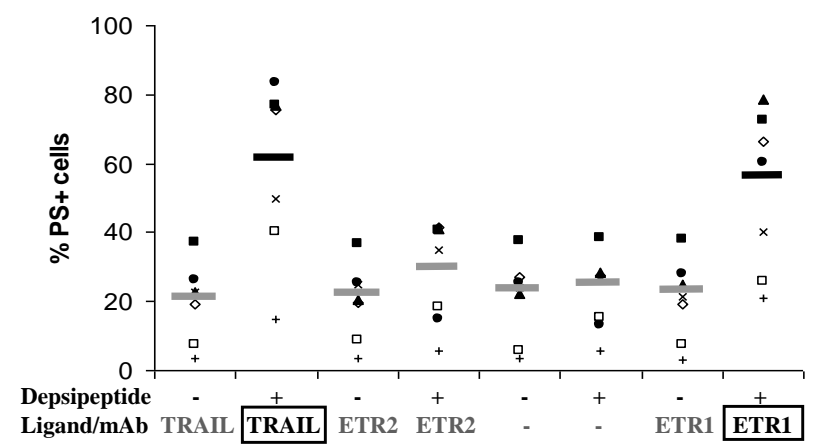

Intriguingly, our data reveal an unanticipated preferential apoptotic signalling via either TRAIL-R1 or TRAIL-R2 in tumour cells (Figure 1, and data not shown). Contrary to literature reports that TRAIL-induced apoptosis occurs primarily via TRAIL-R2, we show that primary CLL cells, in the presence of an HDACi, undergo predominantly TRAIL-R1-mediated apoptosis.

Based on the structure of the TRAIL/TRAIL-R1/R2 complex, we have synthesized mutant forms of TRAIL that specifically trigger apoptosis via -R1 or -R2. Using these mutants, we now demonstrate unequivocally that primary lymphoid tumour cells signal to apoptosis via TRAIL-R1. Thus, no significant therapeutic benefit can be anticipated from treating such patients with TRAIL agents that signal predominantly via TRAIL-R2. Importantly, our data highlight the need to determine whether cells from a particular tumour signal via TRAIL-R1/R2, as such information will help provide a rational approach to optimize TRAIL therapy and also minimize the potential of unwanted toxicity. 